\title{
Effect of a single dose of ibuprofen lysinate before embryo transfer on pregnancy rates in cows
}

\author{
M. Elli ${ }^{1}$, B. Gaffuri ${ }^{2}$, A. Frigerio ${ }^{1 *}$, M. Zanardelli ${ }^{3}$, D. Covini ${ }^{4}$, \\ M. Candiani ${ }^{1}$ and M. Vignali ${ }^{2}$ \\ 1 II Department of Obstetrics and Gynecology, University of Milano, Via Commenda 12, \\ 20122 Milano, Italy; ${ }^{2}$ Istituto Auxologico Italiano, Milano, Italy; ${ }^{3}$ Division of Animal \\ Production, Como, Italy; and ${ }^{4}$ LisaPharma, Erba, Como, Italy
}

Embryo implantation is a critical step in both cows and humans. The use of ibuprofen lysinate to enhance implantation has been investigated in cattle with the specific aim of improving pregnancy rates after embryo transfer. In this study, heifers $(n=100)$ were assigned randomly to one of two groups: one group was treated i.m. with $5 \mathbf{~ m g}$ ibuprofen lysinate $\mathrm{kg}^{-1}$ body weight $1 \mathrm{~h}$ before embryo transfer and a control group received vehicle only. A single embryo was transferred into each recipient cow.
There was a significant difference in the number of pregnancies after embryo transfer between cows in the treated (41 of $50 ; 82 \%)$ and control (28 of $50 ; 56 \%$ ) groups $(P<0.05)$. These data indicate that ibuprofen lysinate may be an effective adjunctive treatment for assisted reproduction in cattle. Further studies are needed to clarify whether this effect is associated with the reduction of cyclooxygenase enzyme isoforms during embryo transfer or whether other mechanisms are involved.

\section{Introduction}

Ibuprofen is a propionic acid derivative non-steroidal antiinflammatory drug that has a number of beneficial actions in addition to its analgesic and antipyretic effects (Breuhaus et al., 1999). These actions have been described for acute and traumatic situations, including injuryinduced immunosuppression, traumatic neurological disease, myocardial ischaemia and various forms of shock (Rockwell and Ehrlich, 1990). Most of the salutary effects of this drug have been attributed to its ability to prevent formation of prostanoids by functional inhibition of both isoforms of cyclooxygenase (COX-1 and COX-2). Moreover, other mechanisms, such as reducing oxidant or cytokine release, scavenging oxygen-derived free radicals and chelating iron have been proposed (Breuhaus et al., 1999).

Reproductive physiology during embryo implantation has been studied widely and the results have been used to improve pregnancy rates in assisted reproduction. In particular, embryo implantation represents a critical step in these techniques in both cows and humans. The implantation rates are about 10 and $50 \%$ per embryo in humans and cows, respectively, with resultant low pregnancy rates that potentially could be improved (Hasler, 1992; Lopata, 1996). Therefore, much effort is directed towards facilitating the crucial phase of embryo implantation.

Recent clinical evidence indicates that administration of substances that inhibit cyclooxygenase enzyme isoforms

*Correspondence

Email: ambrogiofrigerio@libero.it might improve the outcome of in vitro fertilization-embryo transfer cycles. Specifically, Rubinstein et al. (1999) treated a group of women undergoing IVF $(n=149)$ with a daily dose of $100 \mathrm{mg}$ aspirin and obtained significant improvements in implantation and pregnancy rates compared with non-treated women. The pathophysiological significance of molecules derived from cyclooxygenase activity in various female reproductive functions has been studied extensively. These studies provided evidence that the cyclooxygenase pathways are critical for most reproductive events in various species and that the targeted disruption of COX-2, but not COX-1, in mice results in failure of specific processes such as ovulation, fertilization, blastocyst hatching, implantation and decidualization (van der Weiden et al, 1993; Sayre and Lewis, 1993; Dinchuk et al, 1995; Psychoyos et al., 1995; Charpigny et al, 1997; Lim et al., 1997; Nayak et al., 1997; Reese et al., 1999). However, when concentrations of substrates from cyclooxygenase activity that are produced locally at the uterus are increased, endometrial receptivity might be affected adversely (Rubinstein et al., 1999). Prostaglandins stimulate inflammatory cells and stimulate uterine contractions, whereas thromboxane $A_{2}$ induces platelet aggregation and vasoconstriction. Therefore, a successful reproductive event is thought to be dependent on a very delicate equilibrium of the specific mediators generated by cyclooxygenase. In a situation such as embryo transfer, in which the cyclooxygenase pathway is probably highly activated, a drug that reduces the production of these factors may help to avoid the potential negative effects of the cyclooxygenase pathway and consequently improve embryo implantation.

The aim of the present study was to verify this possibility 
in cows. In particular, the effect of a single dose of ibuprofen lysinate, a derivative of ibuprofen with high solubility (Latini et al, 1977), on implantation and pregnancy rates in cycles of embryo transfer in cattle was examined.

\section{Materials and Methods}

In a prospective randomized study, embryo transfer was performed in 100 heifers. Donor Italian Frisona heifers were induced to superovulate by administration of Follitropin ( $\mathrm{FSH}$; Serono, $\mathrm{Mi}$ ) and Cloprostenol $\left(\mathrm{PGF}_{2 \alpha}\right.$; Fatro, Ozzano, Bo) and were artificially inseminated 60 and $72 \mathrm{~h}$ after the injection of $\mathrm{PGF}_{2 \alpha}$. Seven days after first artificial insemination, embryos produced in vivo (Italian Frisona $\times$ Italian Frisona) were recovered by uterine flushings with PBS (Sigma Chemical Company, St Louis, MO) supplemented with $0.04 \%(\mathrm{w} / \mathrm{v})$ BSA fraction $\mathrm{V}$ (Sigma Chemical Company). Embryos were washed, handled in a solution of PBS supplemented with $0.4 \%$ (w/v) BSA fraction $\mathrm{V}$, evaluated for quality grade (excellent: 1 ; good: 2 ) and cryopreserved according to a standard freezing procedure (Niemann, 1983). Recipient cows were selected for embryo transfer on the basis of rectal palpation of the ovaries and uterus and observation of external genitalia. Therefore, on day $7 \pm 1$ of the oestrous cycle of recipient heifers, blastocysts were thawed and transferred nonsurgically into the uterine cavity. The heifers were divided randomly into treatment and control groups. Heifers in the treatment group $(n=50)$ received $5 \mathrm{mg}$ ibuprofen lysinate $\mathrm{kg}^{-1}$ (i.m.) $1 \mathrm{~h}$ before embryo transfer, whereas heifers in the control group $(n=50)$ received vehicle only. Progesterone concentrations on the day of embryo transfer were similar between treated $\left(5.9 \pm 3.3 \mathrm{ng} \mathrm{ml}^{-1}\right)$ and non-treated $\left(6.1 \pm 2.5 \mathrm{ng} \mathrm{ml}^{-1}\right)$ heifers. The recipients were all housed under similar conditions and were fed according to Italian feeding standards for dairy cattle.

Pregnancy status was diagnosed by ultrasonography on day 30 and by both ultrasonography and rectal examination on day 60 after embryo transfer. Finally, rectal palpation was repeated at month 5 of gestation to confirm the presence of fetuses.

The data were analysed by chi-squared test and the level of significance was $P<0.05$.

\section{Results}

A single embryo graded as 'excellent' or 'good' was transferred to each treated and control heifer. Embryos were divided equally into the two groups with regard to quality grade: 26 'excellent' and 24 'good' embryos were transferred to the non-treated heifers and 27 'excellent' and 23 'good' embryos were transferred to the treated heifers. The pregnancy rate as diagnosed by ultrasonography on day 30 after embryo transfer, was significantly higher in the group of heifers treated with ibuprofen lysinate $(82 \%)$ compared with the group that received vehicle $(56 \%)(P<0.05)$ (Fig. 1). As no spontaneous termination of

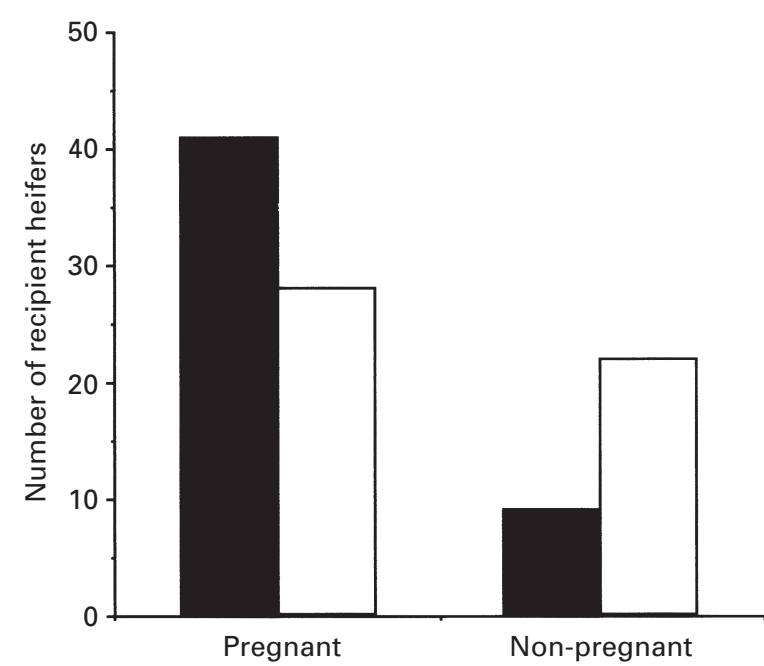

Fig. 1. Outcome of embryo transfer in heifers treated with ibuprofen lysinate ( $\square$ ) and in control heifers $(\square)$ as determined by ultrasonography on day 30 after embryo transfer. Fifty heifers were treated with $5 \mathrm{mg}$ ibuprofen lysinate $\mathrm{kg}^{-1} 1 \mathrm{~h}$ before embryo transfer, whereas heifers in the control group $(n=50)$ received vehicle only. The pregnancy rate was significantly higher in treated heifers than in control heifers $(P<0.05)$.

gestation was evident on day 60 after embryo transfer (as evaluated by both ultrasonography and rectal examination) the pregnancy rates were the same on days 30 and 60 after embryo transfer. When data were analysed in relation to embryo grade, it was observed that ibuprofen lysinate enhanced pregnancy rate equally in heifers into which grade 1 or 2 embryos were transferred (85.19 and $78.26 \%$ for embryos of grades 1 and 2, respectively).

\section{Discussion}

In the present study implantation and pregnancy rates were significantly higher in heifers that received ibuprofen lysinate treatment during cycles of embryo transfer. These results may appear unexpected if it is considered that, among the pharmacological properties of ibuprofen, the most significant effect is the inhibition of cyclooxgenase enzyme isoforms (Breuhaus et al., 1999). Substances derived from cyclooxygenase activity play an important role in endometrial vascularity, blastocyst hatching, embryo implantation and decidualization (van der Weiden et al., 1993; Dinchuk et al, 1995; Psychoyos et al., 1995; Charpigny et al., 1997; Lim et al., 1997; Reese et al., 1999). Indeed, prevention of prostaglandin synthesis through a timed application of a prostaglandin synthesis inhibitor inhibits or delays these processes. Consistent with this observation, administration of DuP697, a selective inhibitor of COX-2 activity, at $\geqslant 600 \mathrm{nmol} \mathrm{I}^{-1}$ on days 3 and 4 of pregnancy resulted in a dose-dependent inhibition of implantation in mice (Lim et al., 1997). Gupta et al. (1981) observed that intrauterine administration of three antiprostaglandin drugs (acetylsalicylic acid, indomethacin 
and ibuprofen) at high doses on day 4 of pregnancy inhibited implantation in rats (Gupta et al., 1981). However, such effects were not evident when ibuprofen was administered at low doses (100 or $200 \mu \mathrm{g}$ per day per animal. Similarly, Najak et al. (1997) reported that suppression of prostaglandin synthesis by high doses of diclofenac during the early- to mid-luteal period in rhesus monkeys resulted in a $75 \%$ inhibition of implantation. Finally, ovine embryos incubated with indomethacin demonstrated a low hatching rate (Sayre and Lewis, 1993).

However, it has been suggested that increased concentrations of locally produced cyclooxygenase-derived mediators may affect implantation of embryos through the activation of inflammatory cells and the stimulation of uterine contractions. Thromboxane $\mathrm{A}_{2}$ in particular has a potent thrombotic effect that may affect implantation by reducing uterine blood flow and tissue perfusion (Rubinstein et al., 1999). These changes would result in a marked constriction of the spiral vessels associated with ischaemic conditions and cellular necrosis (Rubinstein et al., 1999). Prostaglandins stimulate monocytes, lymphocytes, macrophages and neutrophils, thus inducing inflammation (Rubinstein et al., 1999). In this case, the reduction of these adverse effects may actually be beneficial for the establishment of a successful pregnancy. It should also be noted that bovine conceptuses secrete large quantities of trophoblast protein 1, also known as interferon (IFN) $\tau$, between days 17 and 22 of gestation, which acts in an antiluteolytic manner to either inhibit or alter the pattern of endometrial release of $\mathrm{PGF}_{2 \alpha}$ to remove its luteolytic effect (Kerbler et al., 1997). This finding indicates that the inhibition of prostaglandin activity may represent a physiological and necessary event during early pregnancy. In agreement with this hypothesis and with the findings of the present study, treatment with low doses of aspirin improves uterine blood flow velocity, implantation and pregnancy rates in women undergoing IVF (Rubinstein et al., 1999). Aspirin affects blood flow by shifting local production of thromboxane and prostaglandins towards prostacyclin, which has been proposed to modulate the relaxation of vascular smooth muscle of endometrial vessels (Rubinstein et al., 1999). Importantly, embryo transfer is a process that can induce a local inflammatory reaction with increased synthesis of prostaglandins. In this context, the limitation of such an effect by the use of an antiprostaglandin compound during embryo transfer may be favourable to implantation. Ibuprofen in particular has been demonstrated to affect uterine physiology by local inhibition of prostaglandin synthesis (Csapo, 1977; Powell and Chan, 1984; Milson and Andersch, 1985). Therefore, the apparent differences between the results of the present study and those obtained for other species may be related to the dose of the antiprostaglandin used. The dose of ibuprofen administered could be a crucial factor in explaining the discrepancies observed, as high doses of antiprostaglandins are known to induce deleterious rather than beneficial effects. From pharmacokinetic studies performed in rabbits and rats, it seems evident that the dose of ibuprofen administered to cattle in the present study is unable to inactivate cyclooxygenase fully (Csapo, 1977). The aim of the treatment in the present study was not to inactivate cyclooxygenase fully, as the rationale for the therapy was to decrease but not to abolish the production of cyclooxygenase-derived compounds. However, a single administration of a similar dose has been reported to reduce intra-uterine pressure in humans (Milson and Andersch, 1985).

It is also possible that other effects independent of the described potential of ibuprofen as a cyclooxygenase inhibitor may be involved. Ibuprofen lysinate inhibits activation and translocation of the key transcription factor, nuclear factor kappa B (NF-к $\beta$ ). Translocation of this factor from the cytoplasm into the nucleus and the subsequent DNA binding is an essential prerequisite for the upregulation of many pro-inflammatory genes, such as tumour necrosis factor $\alpha$ and interleukin 1 (Stuhlmeier et al, 1999). Moreover, ibuprofen influences the expression of specific adhesion molecules and induces angiogenic-promoting cytokines (Menzel et al., 1999). Thus, further studies are needed to clarify whether the effect of ibuprofen lysinate on pregnancy rate observed in the present study may be due to its ability to affect local expression of cytokines and other molecules important for endometrial function.

It should also be noted that there might be differences among species with respect to the biological efficacy of ibuprofen lysinate. Indeed, there may be a different degree of unidirectional metabolic conversion of one of the two chiral isomers into the other form (Breuhaus et al., 1999). It is thought that the $\mathrm{S}$-form of the ibuprofen enantiomers is responsible for biological activity. A racemic mixture (equal ratio of $\mathrm{R}$ and $\mathrm{S}$ enantiomers) was administered in the present study but the conversion of $\mathrm{R}$ to $\mathrm{S}$ arylpropionic acids, such as ibuprofen, during the absorption phase or in the liver may vary in different species. For example, in humans the ratio of $\mathrm{R}$ to $\mathrm{S}$ enantiomers after administration of a racemic mixture may be $4: 6$, whereas rats are considerably different from humans in that they are incapable of $\mathrm{R}$ to $\mathrm{S}$ unidirectional conversion.

In conclusion, ibuprofen lysinate significantly improves pregnancy rates in cows after embryo transfer and, thus, is likely to be a useful, effective and safe adjunctive treatment for assisted reproduction in cattle.

The authors thank P. Viganò for her interest in this work, critically reviewing the manuscript and her valuable advice.

\section{References}

Breuhaus BA, Graves FJD, Honore EK and Papich MG (1999) Pharmacokinetics of ibuprofen after intravenous and oral administration and assessment of safety of administration to healthy foals American Journal of Veterinary Research 60 1066-1073

Charpigny G, Reinaud P, Tamby JP, Creminon C and Guillomot M (1997) Cyclooxygenase-2 unlike cyclooxygenase- 1 is highly expressed in ovine embryos during the implantation period Biology of Reproduction 57 1032-1040 
Csapo Al (1977) Inhibition of prostaglandin synthesis and contractility in the rabbit and rat uterus by ibuprofen Prostaglandins 13 735-743

Dinchuk JE, Car BD, Focht RJ et al. (1995) Renal abnormalities and an altered inflammatory response in mice lacking cyclooxygenase II Nature 378 406-410

Gupta U, Malhotra N, Varma SK and Chaudhury RR (1981) Effect of intrauterine administration of antiprostaglandin drugs on implantation in the rat Contraception 24 283-288

Hasler JF (1992) Current status and potential of embryo transfer and reproductive technology in dairy cattle Journal of Dairy Science $\mathbf{7 5}$ 2857-2879

Kerbler TL, Buhr MM, Jordan LT, Leslie KE and Walton JS (1997) Relationship between maternal plasma progesterone concentration and interferon-tau synthesis by the conceptus in cattle Theriogenology 47 703-714

Latini R, Villa S, Gerna M, Tognoni G and de Gaetano G (1977) Bioavailability and platelet aggregation inhibitory activity of ibuprofen lysinate. A comparison with ibuprofen International Journal of Clinical Pharmacology 15 492-495

Lim H, Paria BC, Das SK, Dinchuk JE, Langerbach R, Trzaskos JM and Dey SK (1997) Multiple female reproductive failures in cyclooxygenase 2-deficient mice Cel/ 91 197-208

Lopata A (1996) Implantation of the human embryo Human Reproduction 11 175-184

Menzel EJ, Burtscher H and Kolarz G (1999) Inhibition of cytokine production and adhesion molecule expression by ibuprofen is without effect on transendothelial migration of monocytes Inflammation $\mathbf{2 3}$ 275-286

Milson I and Andersch B (1985) Intra-uterine pressure and serum ibuprofen: observations after oral administration of $400 \mathrm{mg}$ ibuprofen to a patient with primary dysmenorrhoea European Journal of Clinical Pharmacology 29 443-446

Nayak NR, Ghosh D, Lasley BL and Sengupta J (1997) Anti-implantation activity of luteal phase mifepristone administration is not mimicked by prostaglandin synthesis inhibitor or prostaglandin analogue in the rhesus monkey Contraception 55 103-114

Niemann H (1983) Theory and practise of deep-freezing cattle embryos DTW Detsch Tierurzt/ Wochenschr 90 109-114

Powell AM and Chan WY (1984) Differential effects of ibuprofen and naproxen sodium on menstrual prostaglandin production in the rat uterine homogenate Prostaglandins Leukotriens Medicine 13 129-137

Psychoyos A, Nikas G and Gravanis A (1995) The role of prostaglandins in blastocyst implantation Human Reproduction 10 30-42

Reese J, Brown N, Paria BC, Morrow J and Day SK (1999) COX-2 compensation in the uterus of COX-1 deficient mice during the preimplantation period Molecular and Cellular Endocrinology 150 23-31

Rockwell WB and Ehrlich HP (1990) Ibuprofen in acute-care therapy Annals of Surgery 211 78-83

Rubinstein M, Marazzi A and deFried EP (1999) Low-dose aspirin treatment improves ovarian blood flow velocity, implantation, and pregnancy rates in patients undergoing in vitro fertilization: a prospective, randomized, double-blind placebo-controlled assay Fertility and Sterility $\mathbf{7 1}$ 825-829

Sayre BL and Lewis GS (1993) Arachidonic acid metabolism during early development of ovine embryos: a possible relationship to shedding of the zona pellucida Prostaglandins 45 557-569

Stuhlmeier KM, Li H and Kao JJ (1999) Ibuprofen: new explanation for an old phenomenon Biochemical Pharmacology 57 313-320

van der Weiden RM, Verdijk RM, Poelmann RE, Helmerhorst FM and Keirse MJ (1993) The influence of indomethacin on the hatching of mouse blastocysts Prostaglandins Leukotrienes Essential Fatty Acids $\mathbf{4 9}$ 683-686

Received 11 April 2000

Accepted 10 August 2000. 